\title{
Composición nutricional y compuestos fitoquímicos de la piña (Ananas comosus) y su potencial emergente para el desarrollo de alimentos funcionales
}

\author{
Nutritional composition and phytochemical compounds of pineapple (Ananas \\ comosus) and its emerging potential for the development of functional foods \\ Gabriel Hernández Ramírez ${ }^{a}$, Edú Ortega Ibarra ${ }^{a, b}$, Ilse Haide Ortega Ibarra ${ }^{a}$
}

\begin{abstract}
:
The pineapple (Ananas Comusus) is one of the most important commercial fruit crops in the world, it is known as the queen of fruits due to its excellent flavor. Pineapple is the third most important tropical fruit in the world after banana and citrus. It is a very consumed and appreciated fruit has obtained popularity over the years due in part to its content in compounds with a beneficial contribution to human health. Pineapple is a rich source of vitamins, minerals, dietary fiber and other important compounds. The group of bioactive compounds consists of phenolic compounds, which include anthocyanins, phenolic acids, stilbenes, tannis and carotenoids, among others, such as bromelain recognized as the most valuable and studied bioactive fruit these compounds can be considered as nutraceuticals due to the preventive effect against oxidative stress, cardiovascular diseases and cancer. It is important to highlight that the pineapple contains characteristic compounds of high value, so that it constitutes a deposit and genetic patrimony to be explored. This review describes the main nutritional characteristics and their involvement in food and health.
\end{abstract}

Keywords:

Pineapple, bioactive compounds, nutraceuticals, bromelain, oxidative stress

\section{Resumen:}

La piña (Ananas Comosus) es uno de los cultivos comerciales de frutas más importantes del mundo, es conocida como la reina de las frutas por su excelente sabor. La piña es la tercera fruta tropical más importante del mundo después del banano y los cítricos. Es una fruta muy consumida y apreciada que ha adquirido popularidad con los años debido en parte a su contenido en compuestos con aporte benéfico para la salud humana. La piña es una rica fuente de vitaminas, minerales, fibra dietética y otros compuestos importantes. El grupo de compuestos bioactivos consiste en compuestos fenólicos, que incluyen antocianinas, ácidos fenólicos, estilbenos, taninos y carotenoides, entre otros como la bromelina reconocida como el ingrediente bioactivo más valioso y estudiado de la fruta, estos compuestos pueden ser considerados como nutracéuticos debido al efecto preventivo contra el estrés oxidativo, las enfermedades cardiovasculares y el cáncer. Es importante resaltar que la piña contiene compuestos característicos de alto valor, de modo que constituye un reservorio y patrimonio genético por explorar. Esta revisión describe a los principales fitoquímicos asociados a la piña y además presenta las propiedades nutracéuticas y su implicación en la alimentación y la salud.

\section{Palabras Clave:}

Piña, compuestos bioactivos, nutracéuticos, bromelina, estrés oxidativo

\section{Introducción}

Para muchos la reina de las frutas y, sin duda para todos, sinónimo del trópico, la piña (Ananas comosus), también conocida como ananá, es una de las cuatro frutas tropicales más importantes, en cuanto a producción se refiere, junto con el mango, el aguacate y la papaya. Su nombre se debe al parecido con las piñas de los pinos y

\footnotetext{
${ }^{a}$ Centro de Investigación en Nutrición y Alimentación de la Licenciatura en Nutrición. Universidad del Istmo, campus Juchitán de Zaragoza, Oaxaca. Gabriel Hernández Ramírez, gabriel.r@bizendaa.unistmo.edu.mx, https://orcid.org/0000-0001-6621-3381; Edú Ortega Ibarra, eoi@bizendaa.unistmo.edu.mx, https://orcid.org/0000-0002-6504-7366; Ilse Haide Ortega Ibarra ihoi@bizendaa.unistmo.edu.mx
} https://orcid.org/0000-0002-1104-2949

${ }^{\mathrm{b}}$ Autor de correspondencia 
al margen de su importancia como fruto, se ha venido cultivando desde hace mucho tiempo 1.

El cultivo de la piña cobra cada vez mayor importancia a nivel mundial y nacional, siendo una de las frutas tropicales más apetecidas por su excelente sabor, sus propiedades culinarias y medicinales. De acuerdo por el Servicio de Información Agroalimentaria y Pesquera (SIAP), esta fruta alcanzó una producción nacional de 945,210 toneladas en el 2017, siendo Oaxaca el segundo principal productor, con un volumen de 130,436 toneladas 2.

La piña forma parte de la familia de las bromelias, éstas son originarias principalmente de Ámerica subtropical y tropical, comprenden aproximadamente 56 géneros y 3000 especies. La piña, Ananas comusus (L.) Merr, es la especie más importante de la familia Bromoliaceae, que es la que se explota con fines comerciales. Los estudios de diversidad sugieren que se originó entre Brasil, norte de Argentina y Paraguay en los bordes meridionales del Amazonas, desde donde se difundió al curso superior del Amazonas, la zona de Venezuela y las Guayanas. El intercambio entre tribus de las frutas más interesantes para el consumo podría haber favorecido su progresiva difusión hacia la América peninsular y el Caribe 1, 3.

\section{Valor nutricional de la piña}

\section{Valor nutricional de la piña}

La fruta de la piña ha sido considerada como una buena fuente de macro y micronutrientes, lo que la convierte en un producto altamente nutritivo y valioso 4 .

La composición nutricional del fruto se puede ver afectada por la variedad o especie, grado de madurez y la región donde se cultive 5 . El principal componente de la piña es el agua, que constituye el $86 \%$ de su peso, mientras que el principal nutriente corresponde a los carbohidratos, aproximadamente el $13 \%$ de su peso y tiene un bajo aporte de lípidos y proteínas a la dieta 6 . Además, esta fruta contiene cantidades considerables de potasio, calcio, magnesio, resaltando un alto contenido de fibra dietética y vitamina $\mathrm{C}$ también es un alimento con bajo contenido en sodio y en grasas. En el caso de la vitamina $\mathrm{C}$, es considerada el principal antioxidante soluble en agua del cuerpo. Según el Departamento de Agricultura de los Estados Unidos (USDA), la piña contiene $13.12 \mathrm{~g}$ de hidratos de carbono por cada $100 \mathrm{~g}$ de fruta que representa el $10.09 \%$ de la cantidad diaria recomendada de carbohidratos (IDR) establecido por la ingesta de referencia dietética de los Estados Unidos. A pesar de que el alto contenido de hidratos de carbono es esencial en la dieta, el tipo de carbohidrato también juega un papel importante en la salud humana 7,8 .

La sacarosa, fructosa y glucosa son los azúcares predominantes en la piña, destacando la sacarosa que se encuentra en niveles más altos que los otros monosacáridos 9. Para la calidad de la piña, el perfil de azúcar juega un papel importante con respecto a su sabor y su aceptabilidad comercial, debido a que la piña es una fruta no climatérica, el nivel de azúcar en esta fruta depende el tiempo de cosecha, por lo tanto, para obtener piña dulce es necesario cosecharlos a medida que se acercan a la etapa madura 10.

Por otro lado, la Administración de Alimentos y Medicamentos de los Estados Unidos (FDA) define una "buena fuente" de macro y micronutrientes como una porción de alimentos que contienen 10 a $19 \%$ de la ingesta adecuada o dosis diaria recomendada para cada nutriente 11. Usando ese estándar, la piña sería considerada como una buena fuente de vitaminas (vitamina $\mathrm{C}$, tiamina y riboflavina) y minerales (potasio, calcio y magnesio) (Tabla 1). Algunos autores incluyen compuestos antioxidantes de la dieta, un grupo de sustancias no tóxicas, como "micronutrientes antioxidantes" pueden tener múltiples funciones en muchos procesos metabólicos y contrarrestar el estrés oxidativo en el cuerpo humano 12. Por lo tanto, la evaluación de su composición de esta fruta ha sido el foco de muchos investigadores en los últimos años 13.

Tabla 1. Composición nutricional de la piña.

\begin{tabular}{|c|c|c|}
\hline Nutrientes & Unidades & Valor por $100 \mathrm{~g}$ \\
\hline Agua & $g$ & 86 \\
\hline Energía & Kcal & 50 \\
\hline Proteína & $g$ & 0.54 \\
\hline Lípidos totales (grasas) & g & 0.12 \\
\hline Carbohidratos & $g$ & 13.12 \\
\hline Fibra dietética total & $\mathrm{g}$ & 1.4 \\
\hline Azúcares totales & $g$ & 9.85 \\
\hline \multicolumn{3}{|l|}{ Minerales } \\
\hline Calcio, (Ca) & $\mathrm{mg}$ & 13 \\
\hline Hierro, (Fe) & $\mathrm{mg}$ & 0.29 \\
\hline Magnesio,( Mg) & $\mathrm{mg}$ & 12 \\
\hline Fósforo, $(\mathrm{P})$ & $\mathrm{mg}$ & 8 \\
\hline Potasio,( K) & $\mathrm{mg}$ & 109 \\
\hline Sodio, (Na) & $\mathrm{mg}$ & 1 \\
\hline Zinc, (Zn) & $\mathrm{mg}$ & 0.12 \\
\hline \multicolumn{3}{|l|}{ Vitaminas } \\
\hline $\begin{array}{l}\text { Vitamina C (Ácido } \\
\text { ascórbico) }\end{array}$ & $\mathrm{mg}$ & 47.8 \\
\hline Vitamina B1 (Tiamina) & $\mathrm{mg}$ & 0.079 \\
\hline $\begin{array}{l}\text { Vitamina B2 } \\
\text { (Riboflavina) }\end{array}$ & $\mathrm{mg}$ & 0.032 \\
\hline Vitamina B3 (Niacina) & $\mathrm{mg}$ & 0.5 \\
\hline Vitamina B6 & $\mathrm{mg}$ & 0.112 \\
\hline Ácido fólico & ug & 18 \\
\hline Vitamina A & IU & 3 \\
\hline $\begin{array}{l}\text { Vitamina E (Alfa- } \\
\text { tocoferol) }\end{array}$ & $\mathrm{mg}$ & 0.02 \\
\hline $\begin{array}{l}\text { Vitamina K } \\
\text { (Filoquinona) }\end{array}$ & ug & 0.7 \\
\hline
\end{tabular}




\section{Lípidos}

Ácidos grasos

saturados totales

Ácidos grasos

g

0.009

moniinsaturados totales

Ácidos grasos

9

0.013

poliinsaturados totales

Fuente: Departamento de Agricultura de los Estados Unidos (USDA) 6.

\section{Composición Fitoquímica de la piña}

Los compuestos bioactivos son constituyentes extra nutricionales, éstos compuestos están asociados con sus efectos positivos en la salud humana, particularmente en enfermedades cardiovasculares, diabetes y cáncer 14. Entre los compuestos bioactivos descubiertos y agrupados según su estructura y función química incluyen carotenoides, flavonoides, fibra dietética, fitoesteroles y moléculas muy activas como el ácido ascórbico (Tabla 2) 15, 19-24. Los principales compuestos bioactivos reportados en la pulpa de piña son los compuestos antioxidantes tales como ácido ascórbico y carotenoides 16 . Además, los subproductos derivados de la piña, como la cáscara, son una fuente importante de fibra dietética y fenoles Unidos 17, también la fruta es rica en bromelina, un complejo de compuestos que incluye proteinasas y otros compuestos bioactivos (Figura 1) 18.

Tabla 2. Compuestos bioactivos en la fruta de la piña.

\begin{tabular}{|c|c|c|c|}
\hline Fuente & $\begin{array}{l}\text { Pulpa } \\
19-22\end{array}$ & Jugo 23 & $\begin{array}{l}\text { Cáscara } \\
17\end{array}$ \\
\hline $\begin{array}{l}\text { Total de vitamina } \\
\mathrm{C}(\mathrm{mg} / 100 \mathrm{~g})\end{array}$ & 47.8 & - & - \\
\hline $\begin{array}{l}\text { Fenoles totales } \\
\text { (mg/100 g de } \\
\text { GAE) }\end{array}$ & 77.5 & 358 & - \\
\hline $\begin{array}{l}\text { Carotenoides } \\
\text { ( } \mu \mathrm{g} / 100 \text { de } \beta- \\
\text { caroteno })\end{array}$ & 42.8 & - & - \\
\hline Fibra dietética (\%) & 2.8 & - & 70.6 \\
\hline $\begin{array}{l}\text { Fenólicos ligados } \\
\text { a la fibra dietética } \\
\text { (mg/100g de GAE } \\
\text { PS) }\end{array}$ & - & - & 2270 \\
\hline
\end{tabular}

Figura 1. Compuestos bioactivos de la piña

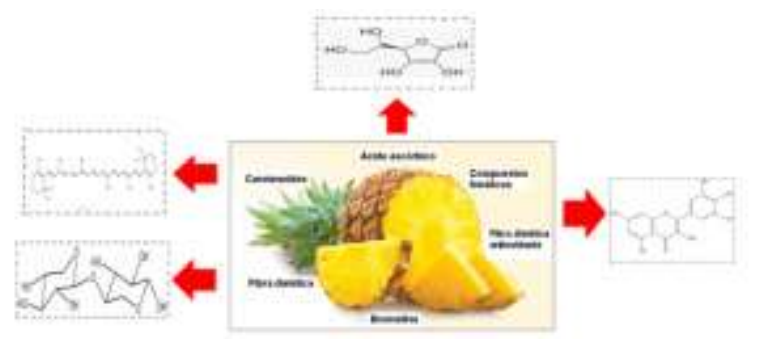

Fuente: Elaboración propia.

\section{El alto valor nutracéutico de la piña}

Los compuestos nutracéuticos asociados a la piña no son necesariamente nutrientes que tradicionalmente han sido asociados con deficiencias en la dieta, sino compuestos cuyo consumo ayuda a prevenir e inclusive tratar a las enfermedades crónico-degenerativas. Estos compuestos se clasifican de acuerdo con sus propiedades químicas y su actividad biológica. Conviene destacar que la diversidad presente entre los compuestos y sus partes en la piña juegan un papel fundamental que incluye la presencia de compuestos bioactivos.

A continuación, se muestran algunos estudios relacionados con la actividad biológica de los compuestos fitoquímicos de la piña (Tabla 3).

Tabla 3. Estudios sobre la actividad biológica de los compuestos fitoquímicos.

\begin{tabular}{|c|c|c|}
\hline Fuente & $\begin{array}{l}\text { Compuestos } \\
\text { fitoquímicos }\end{array}$ & Estudio \\
\hline $\begin{array}{l}\text { Pulpa y } \\
\text { cáscara } \\
\text { de piña } 25\end{array}$ & $\begin{array}{l}\text { Ácido } \\
\text { sináptico, } \\
\text { daucosterol, } \\
\text { 2,5-dimetil- } \\
\text { 4-hidroxi-3- } \\
\text { furanona, } \\
\text { triterpenoide } \\
\text { ergosterol }\end{array}$ & $\begin{array}{l}\text { Los resultados del } \\
\text { estudio in vitro indicaron } \\
\text { que los extractos de } \\
\text { pulpa y cáscara } \\
\text { mostraron potencial } \\
\text { adipogénica, propiedad } \\
\text { anti-glicación y capacidad } \\
\text { protectora al daño DNA, } \\
\text { sugiriendo que los } \\
\text { residuos de piña podrían } \\
\text { utilizarse como } \\
\text { nutracéuticos contra la } \\
\text { diabetes y } \\
\text { complicaciones } \\
\text { relacionada. }\end{array}$ \\
\hline $\begin{array}{l}\text { Residuos } \\
\text { de piña } 26\end{array}$ & Bromelina & $\begin{array}{l}\text { La bromelina purificada } \\
\text { mostró una actividad } \\
\text { antitumoral en líneas de } \\
\text { tumores murinos, siendo } \\
\text { tan activo como el } \\
\text { citostático 5-Flouracilo (5- } \\
\text { FU) frente a modelos } \\
\text { tumorales como la } \\
\text { leucemia (P-388), }\end{array}$ \\
\hline
\end{tabular}




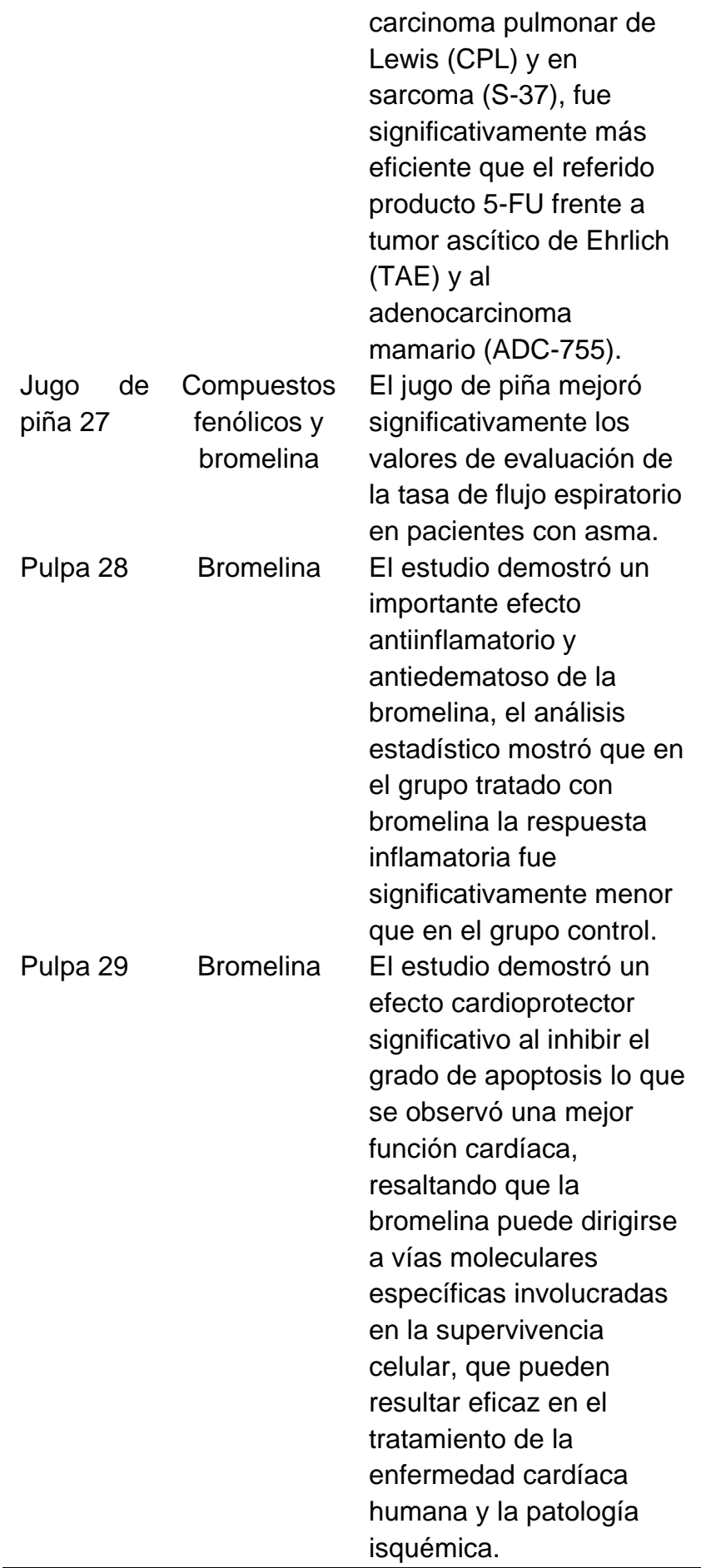

Fuente: Elaboración propia 25-29.

\section{Bromelina}

La piña es una rica fuente de bromelina, una enzima proteolítica, reconocida como el ingrediente bioactivo más valioso y estudiado de la fruta de piña, ha sido investigado desde 1884 y fue identificado por primera vez en 1891 por Marcano 30, 31. La bromelina es un extracto acuoso que contiene una mezcla compleja de proteasas y otras sustancias en pequeñas cantidades. Las enzimas proteolíticas son cisteíno proteasas, ya que un grupo sulfhidrilo libre de una cadena lateral de cisteína se requiere para la actividad enzimática adecuada. Estas enzimas desempeñan un papel importante en la modulación proteolítica de la matriz celular en numerosos procesos fisiológicos 32. La bromelina está presente principalmente en el tallo y una pequeña cantidad presente en subproductos de piña. Las propiedades biológicas de los compuestos de la piña abren una nueva perspectiva para la obtención de productos con valor agregado para el consumo humano.

De acuerdo con lo anterior, se tiene el interés de investigar alimentos que sean fuente de antioxidantes naturales y compuestos bioactivos, ya que éstos no solo proporcionan un beneficio nutricional a los consumidores, sino que, debido a su composición fitoquímica, también ayudan a prevenir o retrasar el desarrollo de padecimientos crónico-degenerativos, dentro de los cuales el cáncer y las enfermedades cardiovasculares son de las más importantes. Tomando en cuenta esto, es necesario desarrollar investigación en alimentos elaborados a partir de la piña, fuente de compuestos biológicamente activos. El interés del consumidor por alimentos que sean saludables proporciona una oportunidad para intensificar la producción de la piña.

Algunas sugerencias generadas a partir de este manuscrito: 1) Promover a nivel del público general las características de interés nutraceútico que la piña pueden proveer en beneficio de la salud; 2) Hacer que la información generada en diversos centros de investigación sea de conocimiento de la industria, y retroalimentar a la academia con las necesidades de ésta; 3) Proponer de manera puntual esquemas sustentables de cómo potenciar su uso regional y nacional; 4) Su consumo debe ser recomendado por diversas organizaciones de la salud.

\section{Conclusión}

La fruta de la piña se puede considerar como una gran fuente de nutrientes, así como de compuestos bioactivos. Por lo tanto, su consumo debe ser recomendado por diversas organizaciones de la salud por sus propiedades funcionales. Del mismo modo es importante crear conciencia sobre el efecto beneficioso de los compuestos fitoquímicos eso se podría obtener por consumo de piña. Hasta ahora, la investigación se ha centrado en su caracterización junto con las tecnologías postcosecha que requiere la piña, sin embargo, es importante promover información sobre la bioaccesibilidad y biodisponibilidad de sus nutrientes y de sus compuestos característicos de la piña que deben considerarse en estudios futuros. La elucidación de los diversos cambios y transformaciones que pueden sufrir los componentes de la matriz alimentaria de la piña mientras se encuentra en el tracto gastrointestinal, pueden proporcionar información valiosa que puede ser aplicada en el desarrollo de nuevos productos como alimentos funcionales, farmacéuticos, así como productos nutracéuticos.

Finalmente, se debe enfatizarse que la piña y sus variedades en México tienen un potencial enorme aún por 
descubrir, por lo que se requiere de investigación para salvaguardar la diversidad de éstas, que incluye la diversidad nutracéutica y la riqueza culinaria que da origen a una parte fundamental de la alimentación de los mexicanos.

\section{Referencias}

[1] Páez, M.G. (1998). Caracterización morfológica de especies silvestres de Ananas spp. Proc. Interamer. Soc. Trop. Hort. Vol. 42:128-132.

[2] Servicio de Información Agroalimentaria y Pesquera. Piña, reina de las frutas tropicales. Servicio de Información Agroalimentaria y Pesquera Blog. Fecha de publicación: 26 de junio de 2018 https://www.gob.mx/siap/articulos/pina-reina-de-las-frutastropicales?idiom $=\mathrm{es}$

[3] Luther, H.E. (2006). An alphabetical list of bromeliad binomials. The Marie Selby Botanical Gardens. Sarasota, Florida, USA. The Bromeliad Society International. $124 \mathrm{pp}$

[4] Valderrain, R.G., Begoña, de A., Sánchez, M.C., González, A.G. (2017). Functional properties of pineapple. Handbook of Pineapple Technology: Production, Postharvest. Science, Processing and Nutrition, (240-257).

[5] Ercisli, S. and Orhan, E. (2007) Chemical composition of white (Morus alba), red ( Morus rubra) and black ( Morus nigra) mulberry fruits. Food Chemistry, 103, 1380-1384. doi:10.1016/j.foodchem.2006.10.054

[6] USDA [United States Department of Agriculture]. National Nutrient Database for Standard Reference Release, [Online], Disponible: http://www.ars.usda.gov/ba/bhnrc/ndl. [Fecha de consulta: 13/04/2019].

[7] Chandalia, M., Garg, A., Lutjohann, D., Von Bergmann, K., Grundy, S.M. Brinkley, L.J. (2000). Beneficial effects of high dietary fiber intake in patients with type 2 diabetes mellitus. New England Journal of Medicine 342(19), 1392-1398.

[8] Lu, X.-H., Sun, D.-Q., Wu, Q.-S., Liu, S., H., Sun, G.-M. (2014). Physico-chemical properties, antioxidant activity and mineral contents of pineapple genotypes grown in China. Molecules Vol. 19(6), 8518-8532.

[9] Lu, X.-H., Sun, D.-Q., Wu, Q.-S., Liu, S., H., Sun, G.-M. (2014). Physico-chemical properties, antioxidant activity and mineral contents of pineapple genotypes grown in China. Molecules Vol. 19(6), 8518-8532.

[10] Cordenunsi, B., Saura-Calixto, F., Diaz-Rubio, M.E., Zuleta, A., Tiné, M.A., Buckeridge, M.S., Silva, G.B.D., Carpio, C., Giuntini, E.B., Menezes, E.W.D. (2010). Carbohydrate composition of ripe pineapple (cv. Perola) and the glycemic response in humans. Food Science and Technology 30(1), 282-288.

[11] Rickman, J.C., Bruhn, C.M. and Barrett, D.M. (2007), Nutritional comparison of fresh, frozen, and canned fruits and vegetables II. Vitamin A and carotenoids, vitamin E, minerals and fiber. J. Sci. Food Agric., 87: 1185-1196. https://doi.org/10.1002/jsfa.2824

[12] Bourassa, M.G. y Tardif, J.C. (2006). Antioxidants and Cardiovascular Disease. USA,Springer.

[13] Farid, H.M., Akhtar, S., Anwar, M. (2015). Nutritional value and medicinal benefits of Pineapple. J Nut and Food Vol. 4, pp. 84-88.

[14] Kris-Etherton, P.M., Hecker, K.D., Bonanome, A., Coval, S.M., Binkoski, A.E., Hilpert, K.F., Griel, A.E. \& Etherton, T.D. (2002). Bioactive compounds in foods: their role in the prevention of cardiovascular disease and cancer. American Journal of Medicine, Vol. 113(9), 71-88.

[15] Sun, J., Chu, Y.-F., Wu, X., Liu, R.H. (2002) Antioxidant and antiproliferative activities of common fruits. Journal of Agricultural and Food Chemistry Vol. 50(25), 7449-7454.
[16] Kongsuwan, A., Suthiluk, P., Theppakorn, T., Srilaong, V., and Setha, S. 2009. Bioactive compounds and antioxidant capacities of phulae and nanglae pineapple. Asian Journal of Food and Agro Industry, 2, 44-50.

[17] Larrauri, J.A., Rupérez, P., Calixto, F.S. (1997). Pineapple shell as a source of dietary fiber with associated polyphenols. Journal of Agricultural and Food Chemistry, Vol. 45(10), 4028-4031.

[18] Maurer, H. (2001) Bromelain: biochemistry, pharmacology and medical use. Cellular and Molecular Life Sciences Vol. 58(9), 1234-1245.

[19] Szeto, Y.T., Tomlinson, B., Benzie, I.F. (2002) Total antioxidant and ascorbic acid content of fresh fruits and vegetables: implications for dietary planning and food preservation. British Journal of Nutrition, Vol. 87(01), $55-59$

[20] Sun, J., Chu, Y.-F., Wu, X., Liu, R.H. (2002) Antioxidant and antiproliferative activities of common fruits. Journal of Agricultural and Food Chemistry Vol. 50(25), 7449-7454.

[21] Silva, L.M.R.D., Figueiredo, E.A.T., Ricardo, N.M.P.S., Vieira, I.G.P., Figueiredo, R.W.D., Brasil, I.M., Gomes, C.L. (2014) Quantification of bioactive compounds in pulps and by-products of tropical fruits from Brazil. Food Chemistry Vol. 143, 398-404.

[22] Ramulu, P. y Udayasekhara Rao, P. (2003) Total, insoluble and soluble dietary fiber contents of Indian fruits. Journal of Food Composition and Analysis, Vol. 16(6), 677-685.

[23] Gardner, P.T., White, T.A., Mcphail, D.B., Duthie, G.G. (2000). The relative contributions of vitamin $\mathrm{C}$, carotenoids and phenolics to the antioxidant potential of fruit juices. Food Chemistry, 68(4), 471-474.

[24] Philip, R.M., Antony, A.K., Vinu, T., Chandrasekharan, C.K., Sasikala, AK. y Gopalan R.K.(2013). An in vitro study reveals nutraceutical properties of Ananas Comosus (L.) Merr. Var. Mauritius fruit reside beneficial to diabetes. J. Sci Food Agric; Vol 94:943-950.

[25] Hernández, M., Carvajal, C., Márquez, M., Báez, R., Morris, H., Santos, R., Chávez, M.A. (2005). Obtención de preparados enzimáticos a partir de tallos de piña (Ananas Comusus) con potencialidades de uso en la Biotecnología y la Medicina. Revista CENIC Ciencias Biológicas. Vol. 36.

[26] Pandit, S. y B. (2015). A study on nutritional efficacy of pineapple juice in the treatment of bronchial asthma. International Journal of Scientific and Research Publications. Vol 5.

[27] Ordesi P, Pisoni L, Nannei P, Macchi M, Borloni R, Siervo S. (2014). Therapeutic efficacy of bromelain in impacted third molar surgery: a randomized controlled clinical study. Quintessence Int; Vol. 45:679-84.

[28] Juhasz, B., Thirunavukkarasu, M., Pant, R., Zhan, L., Varma, S., Secor, E., Srivastava, S., Raychaudhuri, U., Menon, V., Otani, H., Thrall, R., Maulik, Nilanjana. (2008.). Bromelain induces cardioprotection against ischemia-reperfusion injury through Akt/FOXO pathway in rat myocardium. J Physiol Heart Circ Physiol, 294 (3).

[29] Devakate, R., Patil, V., Waje, S., Thorat, B. (2009). Purification and drying of bromelain. Separation and Purification Technology, 64(3), 259264.

[30] Balls, A., Thompson, R., Kies, M. (1941) Bromelin. Properties and commercial production. Industrial and Engineering Chemistry 33(7), 950953.

[31] Paulo A.G. Soares, Antônio F.M. Vaz, Maria T.S. Correia, Adalberto Pessoa, Maria G. Carneiro-da-Cunha, Purification of bromelain from pineapple wastes by ethanol precipitation, Separation and Purification Technology, Volume 98, 2012, Pages 389-395 